Claremont Colleges

Scholarship@ Claremont

All HMC Faculty Publications and Research

HMC Faculty Scholarship

$1-1-2010$

\title{
An Amazing Mathematical Card Trick
}

Arthur T. Benjamin

Harvey Mudd College

\section{Recommended Citation}

Benjamin, A.T. (2010). An amazing mathematical card trick. Math Intelligencer, 32(2): 37-40. DOI: 10.1007/s00283-009-9123-1.

This Article is brought to you for free and open access by the HMC Faculty Scholarship at Scholarship @ Claremont. It has been accepted for inclusion in All HMC Faculty Publications and Research by an authorized administrator of Scholarship @ Claremont. For more information, please contact scholarship@cuc.claremont.edu. 


\section{An Amazing Mathematical Card Trick}

by Arthur T. Benjamin

Harvey Mudd College

A magician gives a member of the audience twenty cards to shuffle. After the cards are thoroughly mixed, the magician goes through the deck two cards at a time, sometimes putting the two cards face to face, sometimes back to back, and sometimes in the same direction. Before dealing each pair of cards into a pile, he asks random members of the audience if the pair should be flipped over or not. He goes through the pile again four cards at a time and before each group of four is dealt to a pile, the audience gets to decide whether each group should be flipped over or not. Then the cards are dealt into four rows of five cards. The audience can decide, for each row, whether it should be dealt from left to right or from right to left, producing an arrangement like the one shown here.

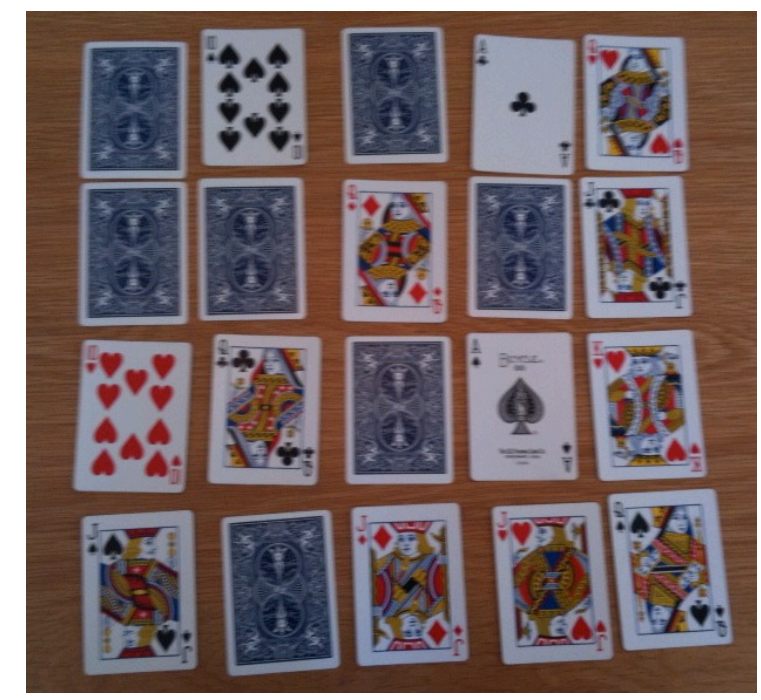

FIGURE 1. A random-looking arrangement of cards.

Finally, the magician "folds" the cards as directed by the audience. For instance, in Figure 1, if the audience requests that the right edge be folded, then this would result in the cards looking like Figure 2. The audience repeats the folding requests until eventually all the cards are in one pile. When the magician spreads the cards, there are fifteen face down cards and five face up cards, and the five face up cards form a royal flush! 


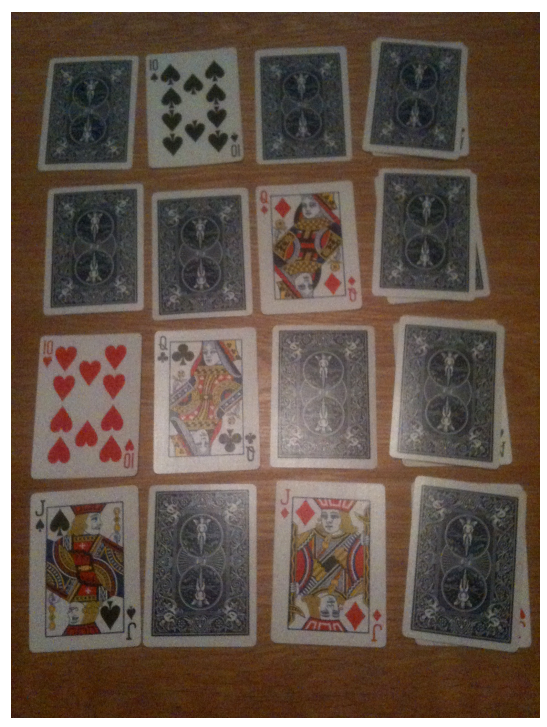

FIGURE 2. After folding the right row, the cards look like this.

As we'll explain, this trick practically works by itself, and the underlying mathematics is simple and elegant. To best explain its secret, we begin with a warmup trick, which is even easier to perform, and also gets a strong audience reaction.

Four Ace Surprise. A magician deals sixteen cards face down into four rows of four cards apiece, then turns four of the cards face up, as in Figure 3.

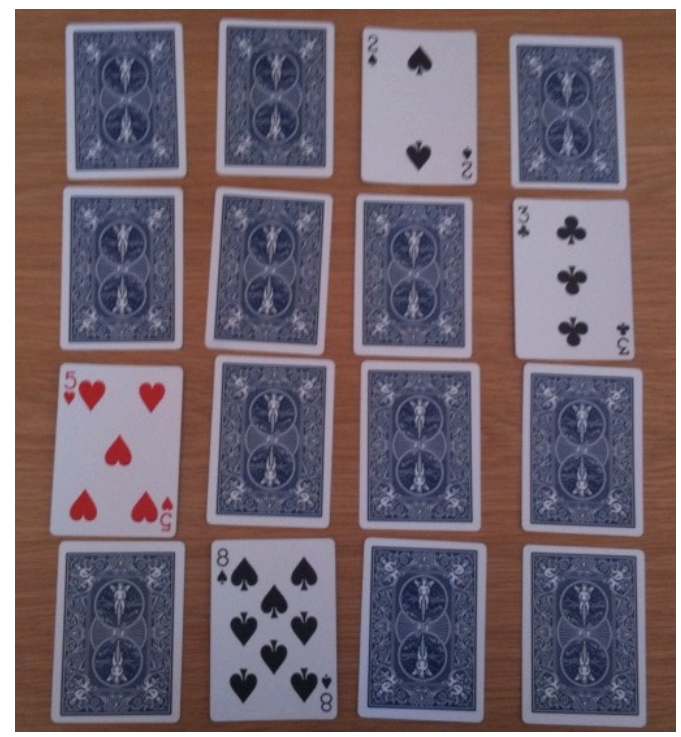

FIGURE 3. The initial layout of cards (with the four aces hidden on the main diagonal)

Next the magician asks the audience to "fold in" the edges of the cards, as in the previous trick. At the end of this procedure, after six folds, all sixteen cards will be stacked into one pile. When the magician spreads the sixteen cards, there will be twelve 
face down cards, and four face up cards. The audience will be surprised to see that the face up cards are the four aces.

The secret. The magician makes sure that the four aces begin on the main diagonal (top left corner to the bottom right corner). To achieve this, the magician could start with the four aces in the first, sixth, eleventh, and sixteenth card in the deck, and then deal the four rows of cards from left to right. An easier (and more deceptive) approach is to stack the four aces at the top of the deck, then haphazardly deal cards into "random" positions in the four-by-four grid, taking care that the aces are placed on the main diagonal. The trick is now guaranteed to work by itself. After all the folding is done, the four aces will be facing one direction and the other twelve cards will be facing the opposite direction. If the aces are face down, then the magician simply turns the stack of cards over before spreading them out.

\section{Why this trick works.}

This trick is a beautiful application of parity. Imagine the sixteen cards are dealt onto a four-by-four checkerboard with black and white squares. In the initial configuration of Figure 2, the white squares are occupied by the four face up cards and the four aces (face down on the main diagonal), and the black squares are occupied by the remaining eight face down cards. After the cards are folded, all the cards will be stacked on a single "destination" square.

Suppose the destination square is black. Now since each ace begins face down on a white square, every ace will be flipped an odd number of times on its way to the destination, and will therefore end face up. By similar reasoning, the four face up cards and the other eight face down cards will be face down at the destination. Likewise, if the destination square is white, then it will contain four face down aces and all other cards will be face up.

Shuffled to your heart's content. We are now ready to explain the trick described at the beginning of the article. It is based on an idea presented in the card magic book, Dear Mr. Fantasy, by John Bannon. In this trick, I use the Ten, Jack, Queen, King, and Ace of each suit from the deck, and ask my assistant to "Please shuffle these twenty cards to your heart's content." After the cards I returned to me, I spread the cards face up and deal the cards, two at a time into a pile on the table. But as I deal the cards, I put some of these pairs face to face, some pairs back to back, and leave some pairs facing the same direction. Next, I deal the cards into four rows of five. The cards look like a random mix of face up and face down cards, like the one shown in Figure 1.

We then go through the folding procedure that was done in the Four Ace Surprise. After all the cards have been folded into a single pile, I say "Now it would be amazing if, after all this shuffling, flipping, and folding, all the cards were now facing the same direction. It didn't happen here, but it would have been amazing. The reason they aren't all in the same direction is because you started this trick by shuffling these cards to your heart's content. And you did quite a nice job of it, because look what we have here..." Next I 
spread the cards to to reveal that there are precisely five face up cards, namely $10 \vee, \mathrm{J}$, $\mathrm{Q} \vee, \mathrm{K} \vee, \mathrm{A} \vee$ (in some order) as I say ".... royal flush in hearts!"

The secret. After the cards are shuffled, and I go through the cards two at a time, each pair has three possibilities:

(i) Neither card is a heart

(ii) Both cards are hearts

(iii) Exactly one card is a heart.

In case (i), the cards are dealt face to face. In case (ii), the cards are dealt back to back. In case (iii), the cards are both dealt face up, but the heart card must go second. With case (iii), if the heart is the first of the face up pair, then deal the two cards as a pair; if the heart is in the second position, then deal the two cards singly. Either way, the heart will be the second card dealt into the pile. The trick now works by itself, just like in the Four Ace Surprise.

Example. Suppose that after the assistant shuffles the cards, the cards appear in the order given in Figure 4. Then after being dealt into a pile, the cards would be in the order given in Figure 5. After being dealt into four rows of five, the cards appear as in Figure 1.

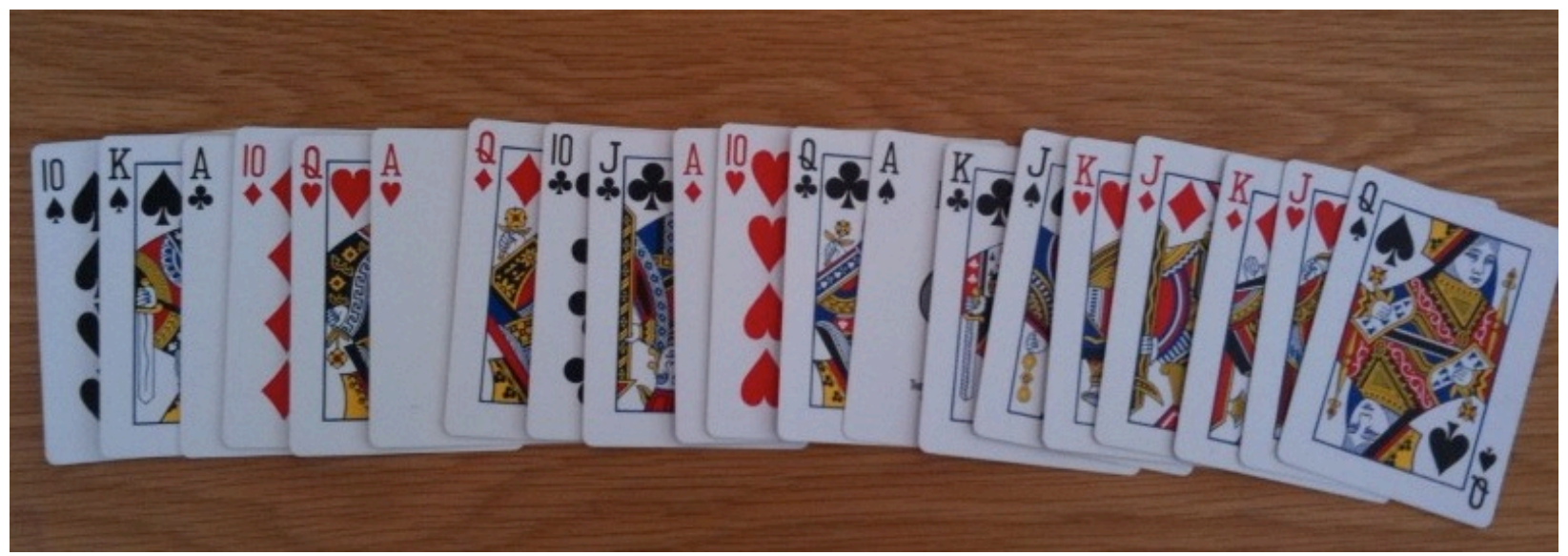

FIGURE 4. The original shuffled twenty cards.

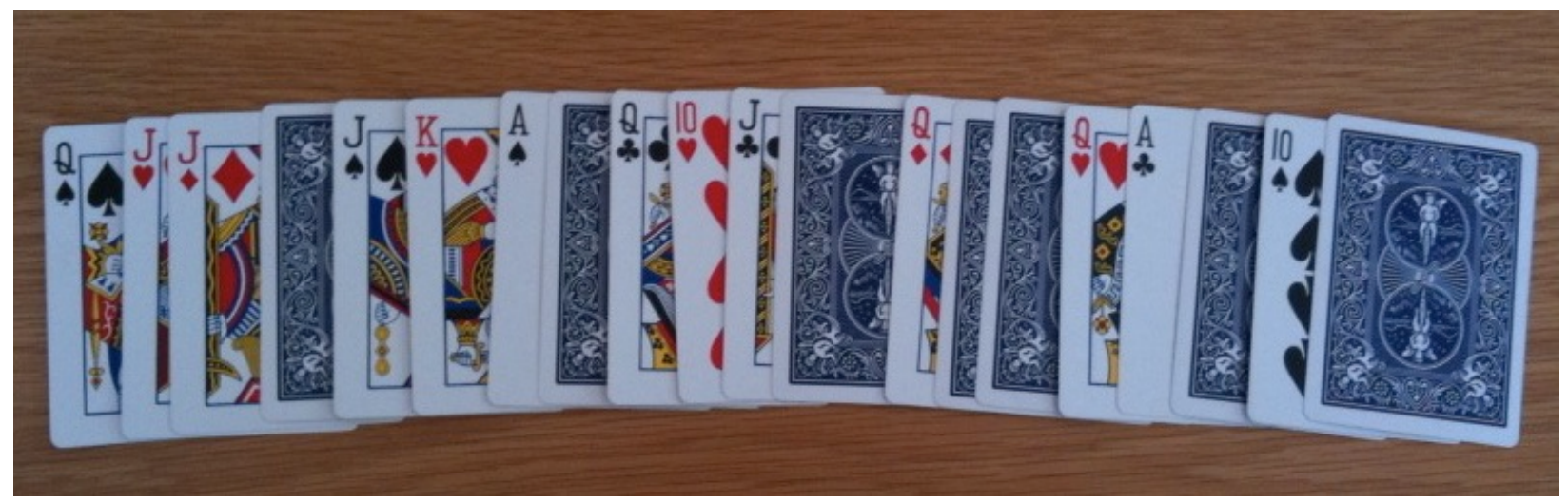


FIGURE 5. The new arrangement after the magician deals the cards, two at a time, into a pile.

Why this trick works. After the shuffled cards are dealt, two at a time, into the pile, let's say that the cards are in positions 1 through 20, as we go from top to bottom. For example, in Figure 5, the King of Spades is face down in position 1, the Ten of Spades is face up in position 2, the Ten of Diamonds is face down in position 3, and so on.

As a result of our dealing rules, regardless of whether we see case (i), (ii), or (iii), the heart cards must either be face up in an odd position or face down in an even position, and the non-hearts have the opposite situation (face down in an odd position or face up in an even position). Notice that this situation remains true even if a pair of cards is flipped over during the deal. Thus, to make the trick more interesting, as I deal each pair of cards, I will ask my assistant (or random members of the audience) if she wants the cards to be flipped or not. After dealing all the cards two at a time, I then go through the cards four at a time, and ask the assistant, for each group of four cards, whether the cards should be flipped or not. It still remains true that the hearts (and only the hearts) will be face up in an odd position or face down in an even position.

Finally, as the magician deals the cards into four rows of five on an invisible checkerboard (with a white square in the upper left corner), each heart will either be face up on a white square or face down on a black square, and the non-hearts will have the opposite situation. In fact, as long as you deal the cards onto the checkerboard so the colors alternate, your hearts and non-hearts will be in the right positions. (I let my assistant decide if she wants the cards dealt left to right, right to left, in zig-zag order, or as a spiral. Note that the trick would not work if you dealt the cards from top to bottom.) As in the Four Ace Surprise, the folding procedure will automatically result in all the hearts facing one direction and the non-hearts facing the other direction.

You can imagine variations of this trick using alphabet cards, number cards, or more complicated instructions. It's a fun trick to perform for any audience, and I enjoy performing it (and sometimes explaining it) for my discrete mathematics class. Either way, this trick is surely one that your audience should flip over.

Acknowledgment. I am grateful to John Bannon for giving me permission to describe his trick in this article.

\section{References.}

The ideas behind this trick were developed and discussed by Henry Dudeney, Bob Hummer, Steve Freeman, Martin Gardner, John Bannon, and Lennart Green, as described in Bannon's book, Dear Mr. Fantasy, published in 2004. For more applications of these ideas, see the online article, Many Fold Synergies, http:// www.maa.org/columns/colm/cardcolm200603.html by Colm Mulcahy. 